\title{
The role of instrument compliance in normal force measurements of polymer melts
}

\section{Journal Article}

Author(s):

Schweizer, Thomas; Bardow, André (D)

Publication date:

2006-04

Permanent link:

https://doi.org/10.3929/ethz-b-000024356

Rights / license:

In Copyright - Non-Commercial Use Permitted

Originally published in:

Rheologica Acta 45(4), https://doi.org/10.1007/s00397-005-0056-0 


\section{Thomas Schweizer André Bardow}

\section{The role of instrument compliance in normal force measurements of polymer melts}

Received: 29 June 2005

Accepted: 5 October 2005

Published online: 8 March 2006

(C) Springer-Verlag 2006

Extended Version of a paper presented at the 2nd Annual European Rheology Conference in Grenoble, France, April 21-25, 2005

T. Schweizer $(\bowtie) \cdot$ A. Bardow Institute of Polymers, ETH Zürich, CH-8093 Zürich, Switzerland e-mail: thomas.schweizer@mat.ethz.ch Tel.: +41-44-6323104

Fax: +41-44-6321412

\begin{abstract}
Schweizer et al. [J Rheol 48 (6):1345-1363, 2004] showed nonlinear step shear rate data for a polystyrene melt $\left(M_{\mathrm{w}}=200 \mathrm{~kg} / \mathrm{mol}, M_{\mathrm{w}} /\right.$ $\left.M_{\mathrm{n}}=1.06, T=175^{\circ} \mathrm{C}\right)$. For different rheometers, cone angles, and sample sizes, the delayed normal force rise observed therein relative to a compliance-free reference $N_{1}$ (from a thermodynamically consistent reptation model) is shown to depend on rheometer compliance characterized by the instrument stiffness $K_{\mathrm{A}}$. $K_{\mathrm{A}}$ can be obtained from mapping $N_{1}$ on the measured $N_{1, \text { meas. }}$ or directly from mechanical contact measurement with
\end{abstract}

a mismatch of $20-30 \%$. The ranking of the stiffnesses found is $K_{\mathrm{A}}(\mathrm{RMS}$ 800) $>K_{\mathrm{A}}(\mathrm{MCR} 300)>K_{\mathrm{A}}$ (ARES LR2). Once $K_{\mathrm{A}}$ is known, $N_{1 \text {,meas. }}$ data can be corrected by solving the ill-posed Volterra equation involved in it. The correction shown for experiments with the 0.15 -rad cone angle gives very good results. The characteristic decay time of the normal force after cessation of flow scales linearly with the axial response time $t_{\mathrm{a}}$ calculated from $K_{\mathrm{A}}$, cone angle, and sample radius. The torque decay time is practically independent of $t_{\mathrm{a}}$.

\section{Introduction}

In a recent publication, Schweizer et al. (2004) showed nonlinear step shear rate data for a polystyrene (PS) melt $\left(M_{\mathrm{w}}=200 \mathrm{~kg} / \mathrm{mol}, M_{\mathrm{w}} / M_{\mathrm{n}}=1.06, T=175^{\circ} \mathrm{C}\right)$ and compared it with simulation data from a thermodynamically consistent reptation model (TCR, Öttinger (1999). For a shear rate of $10 \mathrm{~s}^{-1}$, their viscosity data in Fig. 6 agreed well between experiment and simulation. The first normal stress difference in Fig. 7, however, showed a clear delay of the experimental data to longer times by about one strain unit. It was argued that this delay was due to instrument compliance. This paper clearly proves this point and because measurements free of axial compliance cannot be performed for melts, a method is proposed to correct data when needed.

If nonlinear experiments are performed in cone and plate geometry, the first normal stress arising tends to push the tools apart. This results in a squeeze flow relaxing on a time scale $t_{\mathrm{a}}$ given by (Hansen and Nazem 1975)

$t_{\mathrm{a}}=\frac{6 \pi R \eta}{K_{A} \alpha^{3}}$ with sample radius $R$, viscosity $\eta$, rheometer axial stiffness $K_{\mathrm{A}}$, and cone angle $\alpha$. In their analysis for a Newtonian fluid, the normal force is measured by a spring-type transducer, which allows one of the tools (say the plate) to be displaced a distance $x$ because of its finite stiffness $K_{\mathrm{A}}$. The transducers in the RMS 800 and ARES LR2 used in the present study are both of the force rebalance type and might have a different dynamic behavior. It will be shown, however, that the model is well suited to describe the behavior of all rheometers.

Hansen and Nazem (1975) assume an arbitrary forcing function that displaces the transducer. In the present work, the normal force generated by the fluid $\left(N_{1}\right)$ is the driving force. The dynamic response of the rheometer may then be modeled by a differential equation governing the transducer displacement, which is driven by $N_{1}$ and resisted by the radial squeeze flow between the separating (or approaching) cone and plate tools. The measured normal stress can be defined as $N_{1 \text {,meas. }}=K_{\mathrm{A}} x / \pi R^{2}$, and according to this model, it is governed by

$\frac{t_{\mathrm{a}}}{\tau_{d}} \frac{d}{d t^{*}} N_{1, \text { meas. }}+N_{1, \text { meas. }}=N_{1}$ 
where $\tau_{\mathrm{d}}$ is the fluid relaxation time, and $t^{*}=t / \tau_{\mathrm{d}}$. The solution to Eq. (2) is Eq. (3).

$N_{1, \text { meas. }}(t)=\frac{1}{t_{\mathrm{a}}} \int_{0}^{t} \exp \left(\frac{u-t}{t_{\mathrm{a}}}\right) N_{1}(u) d u$.

Equation (2) clearly shows that for $t_{a} / \tau_{d} \ll 1$, and for $t / t_{a} \gg 1, N_{1 \text {,meas. }}=N_{1}$. However, if $t_{\mathrm{a}}$ is not significantly smaller than $\tau_{\mathrm{d}}$, the measurements will be impaired by finite stiffness effects. Note that the most dominant contribution to $t_{\mathrm{a}}$ in Eq. (1) stems from the cone angle $\alpha$. In the history of rheometers, the strong dependence of normal force response on cone angle was reported quite early (Kaye et al. 1968; Meissner 1972; Gleissle 1976, 1978; Gleissle and Ohl 1990). In Gleissle's (1978) thesis, the influence of the cone angle on the rheological properties of silicon oils and poly(isobutylene) has been extensively studied with a flywheel-and-clutch rheometer, allowing acceleration and deceleration times down to $5 \mathrm{~ms}$. In the present study, the cone angle dependence is quantitatively investigated and extended to include all parameters in Eq. (1).

For this purpose, a step shear rate test at $1 \mathrm{~s}^{-1}$ for 15 shear units followed by relaxation was performed with a well-characterized melt. $t_{\mathrm{a}}$ and thus the ratio $t_{\mathrm{a}} / \tau_{\mathrm{d}}$ could be varied in a wide range by choosing different sets of parameters $R, K_{\mathrm{A}}$ and $\alpha$ in Eq. (1). The time delays of these experiments were quantified by comparing them to a compliance-free reference $N_{1}(t)=N_{1 \text {,TCR }}(t)$ obtained from a TCR developed by Öttinger (1999). The parameters used therein are summarized in Table 1. For a best match with the measured $N_{1, \text { meas. }}(t), N_{1}(t)$ is made compliant using Eq. (3), with the characteristic time $t_{\mathrm{a}}$.

This simple model has the advantage that the only free parameter to bring $N_{1}(t)$ into agreement with the measured $N_{1 \text {,meas. }}$ is the instrument stiffness $K_{\mathrm{A}}$. If the time delay is only due to compliance (squeeze flow), then all experiments performed on one instrument with different cone angles and radii should be describable by one value of $K_{\mathrm{A}}$. However, if the model should be wrong, $N_{1}(t)$ and $N_{1 \text {,meas. }}(t)$ could still be matched in a certain regime by choosing a different $K_{\mathrm{A}}$. Therefore, $K_{\mathrm{A}}$ has to be confirmed by a rheology-independent method and only if the two values thus obtained are within reasonable proximity, can one have sufficient confidence in Eq. (3).

The intent of this publication is to show a simple method to determine total instrument axial stiffness from rheology, to confirm it by mechanical contact measurements, and once this information is known, to show how measured data can be corrected. It is important to make the distinction between the correct, compliance free data $\eta$ and $N_{1}$ (obtainable only from an ideal compliance-free rheometer) and $\eta_{\text {meas. }}$ and $N_{1, \text { meas. }}$, which is the measured data obtained from a rheometer of finite stiffness.

\section{Theory}

As this paper is about the influence of instrument compliance on measured normal stresses, a compliance-free reference has to be defined for quantification.

The ideal response of the melt (PS, $M_{\mathrm{w}}=206 \mathrm{~kg} / \mathrm{mol}$ ) is simulated with the thermodynamically consistent reptation model TCR Öttinger (1999). The application of this model to a similar melt (PS, $M_{\mathrm{w}}=200 \mathrm{~kg} / \mathrm{mol}$ ) was described by Schweizer et al. (2004). By matching the dynamic storage and loss moduli, a reptation time $\tau_{\mathrm{d}}$ of $2 \mathrm{~s}$ is obtained and the prediction of the linear model, $\eta_{0}=0.42 G_{\mathrm{N}} \tau_{\mathrm{d}}$, yields $G_{\mathrm{N}}=171 \mathrm{kPa}$. However, to get a compliance-free standard which best possibly matches experimental steady state data, these two parameters were adapted as $\tau_{\mathrm{d}}{ }^{\prime}=2.2 \mathrm{~s}$ and $G_{\mathrm{N}}{ }^{\prime}=143 \mathrm{kPa} . G_{\mathrm{N}}{ }^{\prime}$ comes from scaling the simulation data to match $\eta^{0}\left(\dot{\gamma}=1 s^{-1}\right) . \quad \eta_{0}{ }^{\prime}=0.42 G_{\mathrm{N}}{ }^{\prime} \tau_{\mathrm{d}}{ }^{\prime}$ then becomes $132 \mathrm{kPas}$, about $10 \%$ lower than $\eta_{0}$. The prediction obtained with the parameters in Table 1 is denominated $\eta$ and $N_{1}$.
Table 1 Characteristics of the fluid and model parameters

\begin{tabular}{llll}
\hline Parameter & Value & Units & Source \\
\hline$M_{\mathrm{w}}$ & 206 & $\mathrm{~kg} / \mathrm{mol}$ & Kisker, Steinfurt, Germany \\
$M_{\mathrm{w}} / M_{\mathrm{n}}$ & 1.06 & - & Kisker, Steinfurt, Germany \\
$M_{\mathrm{e}}$ & 13 & $\mathrm{~kg} / \mathrm{mol}$ & Fetters et al. (1994) \\
$Z$ & 15.8 & - & $M_{\mathrm{w}} / M_{\mathrm{e}}$ \\
$\tau_{\mathrm{e}}$ & 0.377 & $\mathrm{~ms}$ & Schweizer et al. (2004) and shifted $175^{\circ} \mathrm{C} \rightarrow 172^{\circ} \mathrm{C}$ \\
$G_{\mathrm{N}}{ }^{0}$ & 185 & $\mathrm{kPa}$ & $G^{\prime}$ at min (tan $\left.\delta\right)$ \\
$T$ & 172 & ${ }^{\circ} \mathrm{C}$ & \\
$\eta_{0}$ & 144 & $\mathrm{kPas}$ & This paper \\
$\delta_{2}$ & 0 & - & Intensity of convective constraint release \\
$G_{\mathrm{N}}{ }^{\prime}$ & 143 & $\mathrm{kPa}$ & Fit to exp. data \\
$\tau_{\mathrm{d}}{ }^{\prime}$ & 2.2 & $\mathrm{~s}$ & Fit to exp. data \\
$\tau_{\mathrm{s}}$ & 95 & $\mathrm{~ms}$ & $\tau_{\mathrm{e}} \cdot Z^{2}$ \\
$\lambda_{\max }$ & 4.14 & - & Max chain stretch, procedure of Likhtman and McLeish $(2002)$ \\
\hline
\end{tabular}


Table 2 Comparison of steady state values at $\gamma=1 s^{-1}$

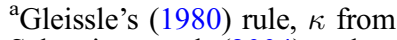
Schweizer et al. (2004) and Schweizer (2002)

${ }^{\mathrm{b}}$ At $\gamma=8.5$, the beginning of steady state viscosity

\begin{tabular}{|c|c|c|c|c|}
\hline Method & Equation & $\eta_{\mathrm{ss}}(\mathrm{kPas})$ & $N_{1, \text { ss }}(\mathrm{kPa})$ & $-N_{2, \text { ss }}(\mathrm{kPa})$ \\
\hline $\begin{array}{l}\text { TCR model } G_{\mathrm{N}}{ }^{\prime}=143 \mathrm{kPa}, \\
\tau_{\mathrm{d}}^{\prime}=2.2 \mathrm{~s}, \delta_{2}=0\end{array}$ & & $70.8 \pm 0.2$ & $122.4 \pm 0.3$ & $31.5 \pm 0.3$ \\
\hline $\begin{array}{l}\text { Experiment RMS } 800 \text {, partitioned plate, } \\
R_{\mathrm{i}}=6 \mathrm{~mm}, \alpha=0.148 \mathrm{rad}\end{array}$ & & $70.8 \pm 0.6$ & $135.4 \pm 7.5^{\mathrm{b}}$ & $14.3 \pm 5^{\mathrm{b}}$ \\
\hline$\Psi_{1, \mathrm{H}}^{0}(t=\kappa / \dot{\gamma}), \kappa=2.2^{a}$ & 3 & & 143.3 & \\
\hline$\eta_{, \mathrm{H}}^{0}(t=1 / \dot{\gamma})$ & & 69.7 & & \\
\hline$\Psi_{1, G}^{0} *(t=\kappa / \dot{\gamma}), \kappa=2.2^{a}$ & 4 & & 158.7 & \\
\hline$\eta_{, G}^{0} *(t=1 / \dot{\gamma})$ & & 72.5 & & \\
\hline
\end{tabular}

A second compliance-free reference is the zero shear rate limit of the first normal stress coefficient $\Psi_{1}^{0}(t)$ calculated with Lodge's theory (Lodge and Meissner 1972) from the relaxation time spectrum $H(\tau)$ :

$\Psi_{1, H}^{0}(t)=2 \int_{0}^{\infty} H(\tau) \tau\left\{1-\left(1+\frac{t}{\tau}\right) \exp \left(-\frac{t}{\tau}\right)\right\} d \tau$

This curve yields the initial slope of $N_{1}(t)$ and the steady state value $\left(\Psi_{1, \mathrm{ss}}\right)$ from Gleissle's rule (see Table 2$)$. It was found that $\Psi_{1}^{0}(t)$ can be calculated equally well with the following relation, without the transition via the spectrum:

$\Psi_{1, \mathrm{G}^{*}}^{0}(t)=\left.\frac{2 G^{\prime}(\omega)}{\omega^{2}}\right|_{t=1.5 / \omega}$
This relation has been verified by the authors for a variety of polymers, with different dispersities and topologies. The steady state values obtained from $N_{1}(t)$ and Eqs. (4) and (5) are summarized in Table 2.

\section{Experiment}

The fluid used in this investigation is a polystyrene melt PS 206k with narrow molecular weight distribution to have a material with a narrow relaxation time spectrum. Characteristic data of the sample can be found in Table 1. A different temperature was chosen compared to the original paper (Schweizer et al. 2004) because the old sample was used up and the new one was originally supposed to have a lower molecular weight than the one finally determined by gel permeation chromatography.

Table 3 Characteristics of rheometers used

\begin{tabular}{|c|c|c|c|c|c|c|c|c|c|}
\hline Name & Manufacturer & $\begin{array}{l}\text { Year of } \\
\text { construction }\end{array}$ & $\begin{array}{l}\text { Maximum } \\
\text { normal } \\
\text { force }(\mathrm{N})\end{array}$ & $\begin{array}{l}\text { Optimum } \\
\text { force } \\
\text { resolution } \\
\text { at } 172^{\circ} \mathrm{C} \\
(\mathrm{mN})\end{array}$ & $\begin{array}{l}\text { Cone angles } \\
\text { used in this } \\
\text { study and } \\
\text { tool diameter } \\
(\mathrm{rad} / \mathrm{mm})\end{array}$ & $\begin{array}{l}\text { Temperature } \\
\text { control }\end{array}$ & $\begin{array}{l}\text { Maximum } \\
\text { temperature } \\
\text { fluctuation on a } \\
\text { time scale of } \\
\text { minutes at } 172^{\circ} \mathrm{C} \\
\left({ }^{\circ} \mathrm{C}\right)\end{array}$ & $\begin{array}{l}\text { Instrument } \\
\text { stiffness } \\
K_{\mathrm{A}} \text { from } \\
\text { rheology } \\
(\mathrm{N} / \mu \mathrm{m})\end{array}$ & $\begin{array}{l}\text { Instrument } \\
\text { stiffness } K_{\mathrm{A}} \\
\text { measured } \\
\text { mechanically in } \\
\text { plate-plate contact } \\
\text { mode }(\mathrm{N} / \mu \mathrm{m})\end{array}$ \\
\hline $\begin{array}{c}\text { RMS } \\
800\end{array}$ & Rheometrics & 1989 & 20 & 2 & $\begin{array}{c}0.1005 / 50 \\
0.148 / 50\end{array}$ & $\begin{array}{l}\text { Both tools elec- } \\
\text { trically heated, } \\
\text { see Meissner } \\
\text { et al. (1989) }\end{array}$ & $\pm 0.005^{\mathrm{a}}$ & 5 & $3.5 \pm 0.13$ \\
\hline $\begin{array}{c}\text { MCR } \\
300\end{array}$ & Paar Physica & 2002 & 50 & 40 & $0.1047 / 50$ & $\begin{array}{l}\text { Electrically } \\
\text { heated plate, } \\
\text { indirectly } \\
\text { heated cone } \\
\text { (hood oven) }\end{array}$ & $\pm 0.01^{\mathrm{b}}$ & 4 & $2.86 \pm 0.03$ \\
\hline $\begin{array}{l}\text { ARES } \\
\text { LR2 }\end{array}$ & $\begin{array}{l}\text { TA } \\
\text { Instruments }\end{array}$ & 2004 & 20 & 10 & $\begin{array}{r}0.04 / 25 \\
0.1 / 25 \\
0.15 / 25\end{array}$ & $\begin{array}{l}\text { gas convection } \\
\text { oven }\end{array}$ & $\pm 0.05^{\mathrm{c}}$ & 2 & $1.69 \pm 0.03$ \\
\hline
\end{tabular}

${ }^{a}$ Measured in center of silver plate, $3 \mathrm{~mm}$ below surface, Pt100 resistor

${ }^{\mathrm{b}}$ Measured in PS melt squeezed between cone and plate, Pt100 resistor

${ }^{\mathrm{c}}$ Measured $1 \mathrm{~mm}$ below plate surface with spring-loaded Pt100 sensor, as displayed by Orchestrator software 


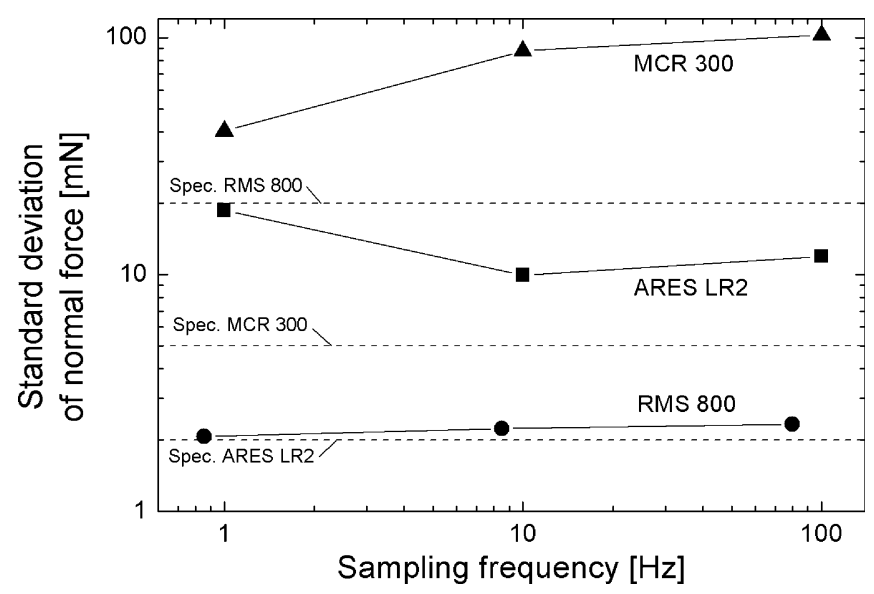

Fig. 1 Force resolution of rheometers. Cone angle approximately $0.1 \mathrm{rad}$, sample HDPE $6011 \mathrm{~L}$ at $150^{\circ} \mathrm{C}, \eta_{0}=21.1 \mathrm{kPas}$, radius $\mathrm{R}$ approximately $13 \mathrm{~mm}$. The data show the standard deviation of 350 data points acquired with the sampling rate shown without any flow. For the specification, a resolution of 1:10,000 is assumed for the ARES LR2 and MCR 300, and 1:1000 for the much older RMS 800

Tablets for rheometry were produced from the precipitated PS powder. Cold-pressed tablets were molten under vacuum as described in Schweizer et al. 2004. The typical sample mass ranged from 0.02 to $0.2 \mathrm{~g}$, so that the radii of the melt squeezed between cone and plate lay between 5 and $12 \mathrm{~mm}$. Three rheometers have been used to dispose of different instrument stiffnesses $K_{\mathrm{A}}$. The characteristics of the three rheometers are summarized in Table 3. Figure 1 shows their force resolution. Without flow, 350 data points of the normal force were collected in three time windows of 3.5-, 35-, and 350-s durations respectively. The standard deviation of the average of these 350 data points measures two quantities: the base line stability of the force measuring device and the influence of the fluctuations from the temperature controller. Only for the RMS 800 is the measured force resolution better than the specifications. For the ARES, the specified resolution is easily achieved at room temperature, but not at measuring temperature $\left(172^{\circ} \mathrm{C}\right)$ with the convection oven. For the MCR 300, the noise of normal force data strongly depends on data averaging by the measuring software. Therefore, the number of data points over average was set to "automatic" for the measurements in Figs. 1 and 2, assuming that this yields an optimized result. The different values for the axial response time $t_{\mathrm{a}}\left(K_{\mathrm{A}}, R, \alpha\right)$ are calculated in Table 4, together with the graph number were the curves appear. Note that only for four configurations is $\tau_{\mathrm{d}}{ }^{\prime}$ at least $50 \%$ larger than $t_{\mathrm{a}}$ (bold numbers).

In each instrument, the sample was protected by high purity nitrogen (evaporated $\mathrm{LN}_{2}$ ). In the ARES convection oven, the heating is via hot nitrogen. In the RMS 800, the electrically heated tools are surrounded by a housing flushed with $5 \mathrm{l} / \mathrm{min}$ of preheated $\mathrm{N}_{2}$. In the MCR 300, $5 \mathrm{l} / \mathrm{min}$ of ambient temperature nitrogen are blown into the hood oven. The plate is directly heated, the cone indirectly. The set-point for the cone heating is determined in such a way that the thermal gradient across the gap is minimal (see Schweizer 2005 for details).

After loading a solid sample to the plate with the help of a centring gauge and penetrating the cone, it was left to relax and thermally equilibrate for $20 \mathrm{~min}$. The radius $R$ of the molten squeezed sample was calculated from its mass $m$ through

$R=\sqrt[3]{\frac{3 m}{2 \pi \alpha \rho}}$

with the cone angle $\alpha$ and the density at measuring temperature $\rho\left(=0.988 \mathrm{~g} / \mathrm{cm}^{3}\right)$.
Fig. 2 Shear rate of $1 \mathrm{~s}^{-1}$ applied during $15 \mathrm{~s}$ to a polystyrene melt with $M_{\mathrm{w}}=206 \mathrm{~kg}$ $\mathrm{mol}^{-1}, M_{\mathrm{w}} / M_{\mathrm{n}}=1.06, T=172^{\circ} \mathrm{C}$ Symbols are averages of two measurements of $N_{1, \text { meas.; }}$; broken lines, $N_{1, \text { meas. }}$ calculated with Eq. 3. $N_{1}, \Psi_{1, \mathrm{G}^{*}}^{0}$ and $\Psi_{1, \mathrm{H}}^{0}$ are the compliance-free references. All data for one cone angle of $\alpha$ approximately $0.1 \mathrm{rad}$. The three groups of $N_{1 \text {,meas. }}$-data are for three different rheometers as shown in the legend: $K_{\mathrm{A}}=4 \mathrm{~N} \mathrm{\mu m}^{-1}$, MCR $300 ; K_{\mathrm{A}}=2 \mathrm{~N}^{-1}$, ARES LR2; $K_{\mathrm{A}}=5 \mathrm{~N} \mathrm{~mm}^{-1}$, RMS 800

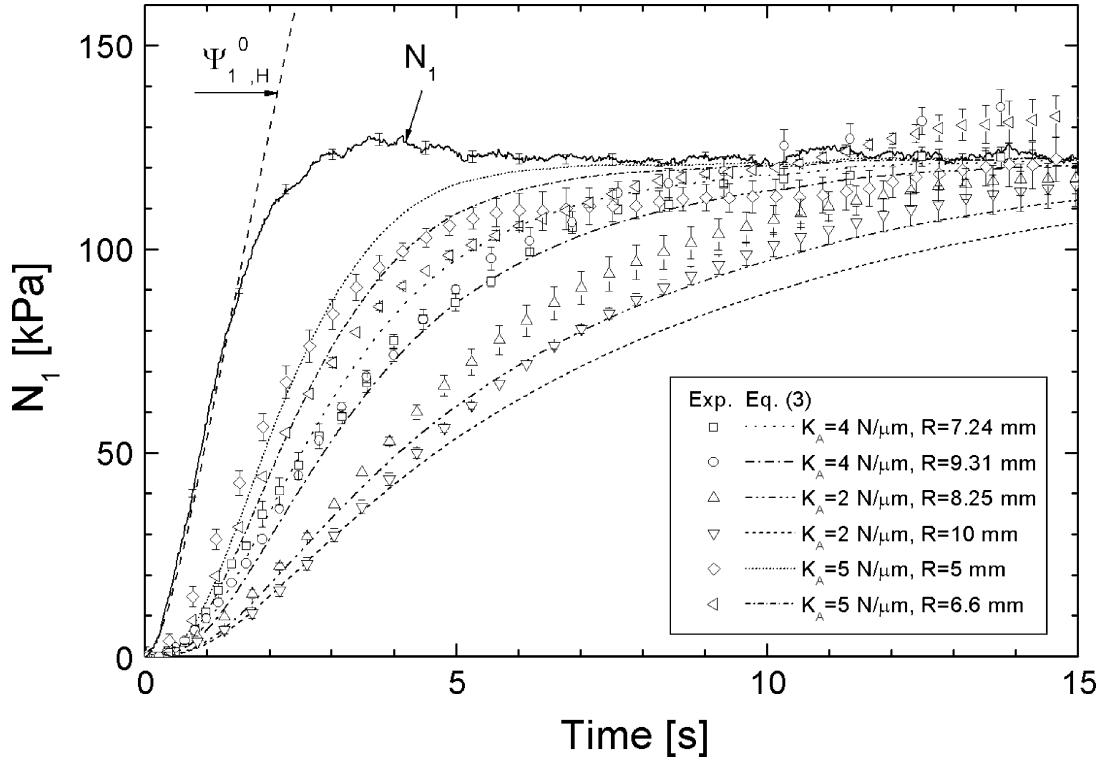


Table 4 Axial response time $t_{\mathrm{a}}$ for rheometers and geometries used

\begin{tabular}{lllllll}
\hline Figure & $\begin{array}{l}K_{\mathrm{A}} \\
(\mathrm{N} / \mu \mathrm{m})\end{array}$ & Instrument & $\begin{array}{l}\text { Cone angle } \\
(\mathrm{rad})\end{array}$ & $\begin{array}{l}\text { Radius } \\
(\mathrm{mm})\end{array}$ & $t_{\mathrm{a}}(\mathrm{s})$ & $\tau_{\mathrm{d}}{ }^{\prime} / t_{\mathrm{a}}(-)$ \\
\hline 4 & 2 & ARES LR2 & 0.04 & 6.9 & 72.1 & 0.031 \\
4 & 2 & ARES LR2 & 0.04 & 8 & 83.6 & 0.026 \\
2,4 & 2 & ARES LR2 & 0.1 & 8.27 & 5.53 & 0.4 \\
2,4 & 2 & ARES LR2 & 0.1 & 10 & 6.69 & 0.33 \\
4,7 & 2 & ARES LR2 & 0.15 & 6.42 & 1.27 & $\mathbf{1 . 7}$ \\
4,7 & 2 & ARES LR2 & 0.15 & 8.33 & 1.65 & 1.3 \\
2,5 & 5 & RMS 800 & 0.1 & 5 & 1.32 & $\mathbf{1 . 7}$ \\
2,5 & 5 & RMS 800 & 0.1 & 6.6 & 1.75 & 1.3 \\
5,7 & 5 & RMS 800 & 0.148 & 6.38 & 0.527 & $\mathbf{4 . 2}$ \\
5,7 & 5 & RMS 800 & 0.148 & 9.2 & 0.76 & $\mathbf{2 . 9}$ \\
2 & 4 & MCR 300 & 0.105 & 7.24 & 2.09 & 1.05 \\
2 & 4 & MCR 300 & 0.105 & 9.31 & 2.69 & 0.82 \\
\hline
\end{tabular}

\section{Results and discussion}

In this section, results are presented in two blocks: First, it is shown that the observed time delay in the transient first normal stress difference can be described by a simple oneparameter model, containing time $t_{\mathrm{a}}$ (Eq. 1) as the characteristic time and that $K_{\mathrm{A}}$ thus obtained is with sufficient accuracy the same as the one measured mechanically. Second, it is demonstrated how well and within which limits normal force data can be corrected for compliance effects. This correction involves axial stiffness $K_{\mathrm{A}}$ and the steady state viscosity at the shear rate concerned as the only parameters.

\section{Viscosity}

Although this work is on normal forces, a few words should be lost about viscosity $\eta_{\text {meas. }}$. It is generally lower than the simulated $\eta(t)$. The reason for this is beyond doubt the disintegration of the sample's rim due to edge fracture. This is evident from two observations: (1) The viscosity is correctly measured within 5\% with the ARES LR2 and a cone angle of $0.04 \mathrm{rad}$. As Fig. 3 shows, the rim is not fractured for this sample. (2) Tests have been made with the cone-partitioned plate geometry (CPP, Schweizer 2002) on the RMS 800 with a radius of the stem $R_{\mathrm{i}}=6 \mathrm{~mm}$. This geometry allows excluding the fracturing rim from the measuring volume. For $R>9 \mathrm{~mm}$, a reproducible $\eta(t)$ with a steady state is measured, which coincides with $\eta(t)$. For $R<9 \mathrm{~mm}$, the fracturing rim and the loss of sample volume by the formation of rolls manifests by the continuous decay of the viscosity as seen in all experiments with cone-plate geometry and $\alpha \geq 0.1 \mathrm{rad}$.

Modelling of the time delay

Figure 4 compares data for the ARES LR2 for three different cone angles. The averages of two repetitive tests with the same geometry are shown. As expected, $N_{1 \text {,meas. }}(t, R, \alpha)$ covers a wide range of values because of the $\alpha^{3}$-dependence in Eq. (1). It should be repeated that all this data is for the same test protocol with a shear
Fig. 3 Photographs of samples recovered after 15 shear units to show the influence of the sample rim height $(\alpha \cdot R)$ on edge fracture

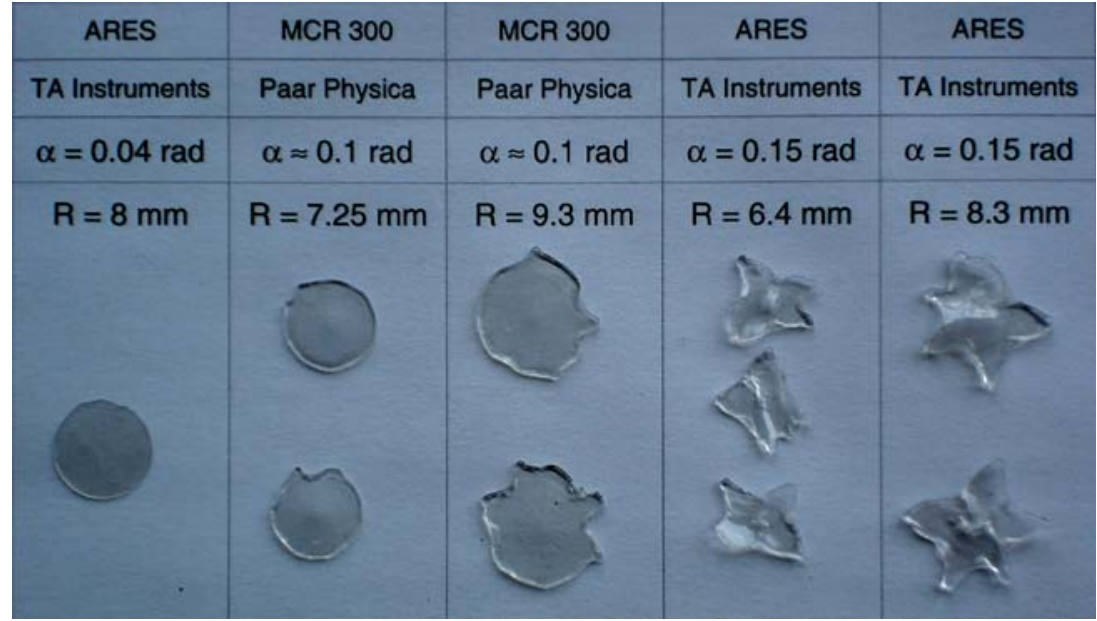


Fig. 4 Same melt and same test as in Fig. 2. Rheometer ARES LR2. The three groups of $N_{1, \text { meas. }}$-data are for three different cone angles as shown in the legend

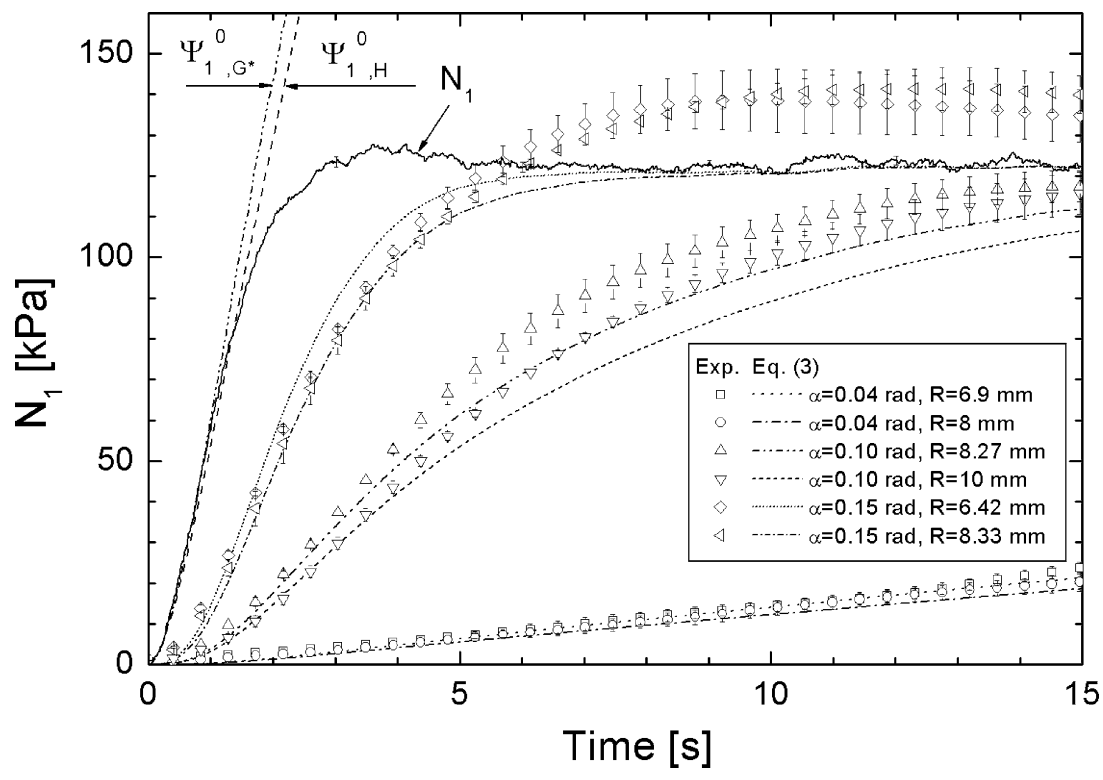

rate of $1 \mathrm{~s}^{-1}$ ! The comparison with the reference curve $N_{1}(t)$ shows that all experimental data is too low. The reproducibility increases with decreasing cone angle. This is due to the sample better keeping its shape for small cone angles, as shown by the photographs in Fig. 3 and as discussed for the viscosity in the preceding paragraph. The simulation data made compliant by Eq. (3) $\left[N_{1} \rightarrow N_{1 \text {, meas. }}(t)\right]$ gives a good description of the experiment in the first few seconds for an instrument stiffness $K_{\mathrm{A}}=2 \mathrm{~N} / \mu \mathrm{m}$.

The angle of $0.1 \mathrm{rad}$ is the only one common to all three rheometers. Data for this cone is shown in Fig. 2 comparing the three instruments. The situation is less dramatic than in Fig. 4, but there is a clear ranking in terms of stiffness:
$K_{\mathrm{A}}(\mathrm{RMS} 800)>K_{\mathrm{A}}(\mathrm{MCR} 300)>K_{\mathrm{A}}(\mathrm{ARES}$ LR2). Again, the model, Eq. (3), can describe the experimentally observed time delay quite well, with a specific $K_{\mathrm{A}}$ for each instrument.

In Fig. 5 finally, the RMS 800 data of the previous picture is compared with the best possible case (in terms of axial response time), namely the RMS 800 with the largest cone angle of $0.148 \mathrm{rad}$. This $N_{1 \text {,meas. }}(t)$ shows a fairly small time deviation from the stiff transient $N_{1}$, which however is still in the order of half a strain unit. Figure 5 also confirms the view of Fig. 3: The strongly fractured edge does not allow to measure the steady state of $N_{1 \text {,meas. }}(t)$ reproducibly.
Fig. 5 Same melt and same test as in Fig. 2. Rheometer RMS 800 . The two groups of $N_{1 \text {,meas. }}$ -data are for two different cone angles as shown in the legend

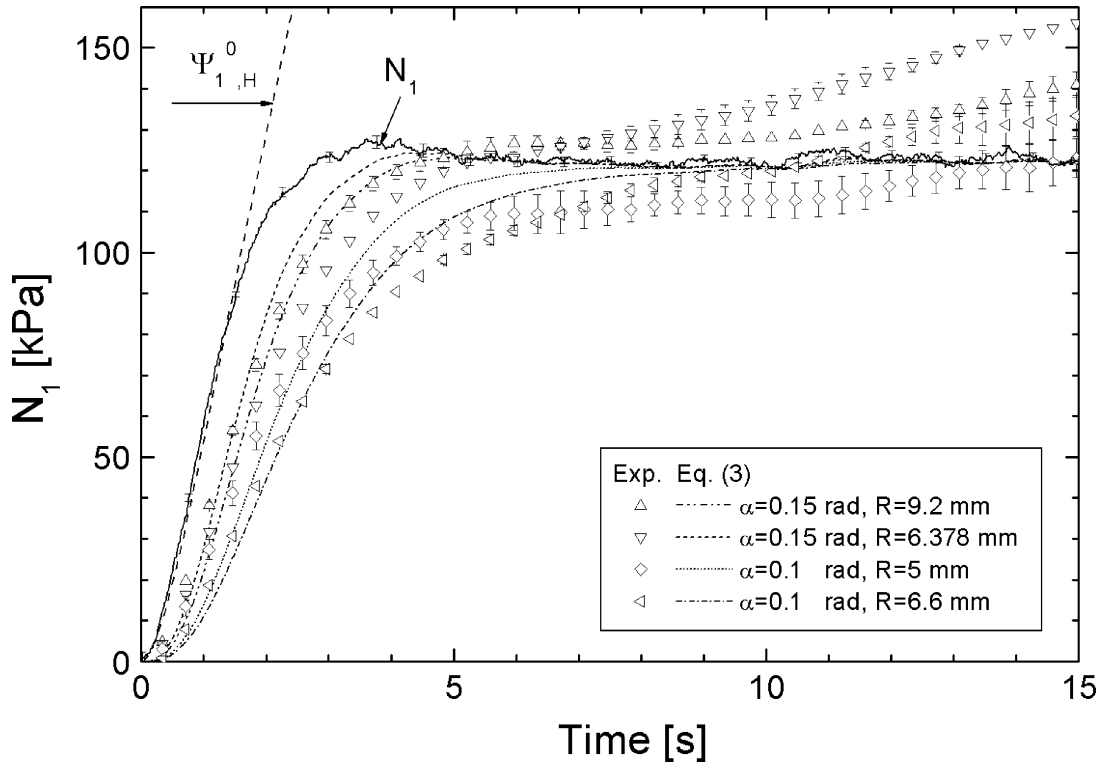




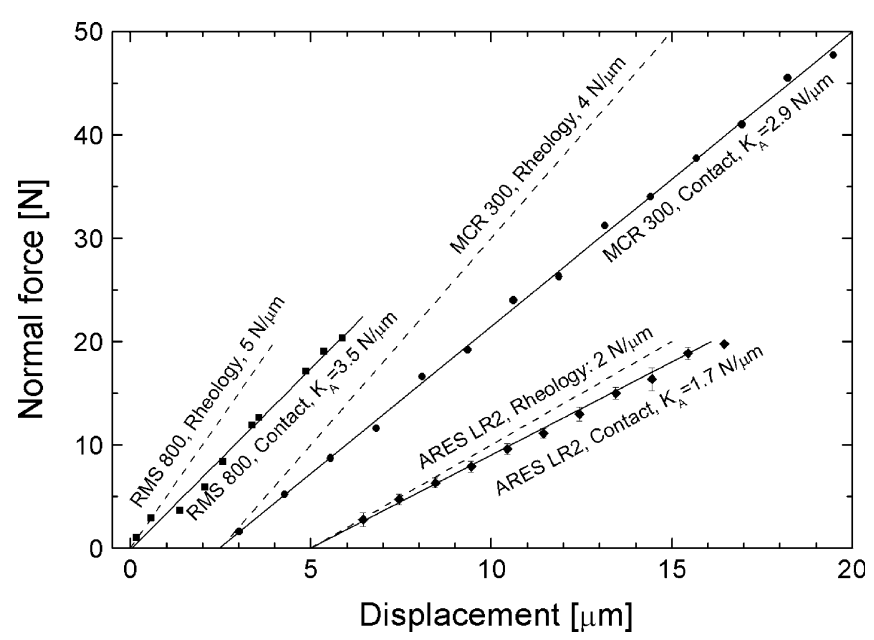

Fig. 6 Comparison of rheometer stiffnesses determined from mechanical contact measurements (symbols and solid lines) and from bringing $N_{1}$ into agreement with $N_{1, \text { meas. }}$ (dashed lines). The groups of curves for the different rheometers have been shifted along the abscissa for better readability

The instrument stiffnesses obtained from matching $N_{1, \text { meas. }}$ $(t)$ with $N_{1}(t)$ in Figs. 2, 4 and 5 are shown in Fig. 6 as dashed lines. This $K_{\mathrm{A}}$ is compared to a $K_{\mathrm{A}}$ measured mechanically by bringing the plate-plate geometry in direct contact, then further reducing the gap and reading off the resulting normal force. By doing so, it is assumed that the instrument frame deformation from the sample's normal force is similar to the one from moving the translation stage of the upper tool-head in plate-plate contact mode. For the RMS 800, the built-in micrometer could not be used, as it measures the displacement between the upper tool-head and the nearby frame and therefore not the full deformation of the frame. A micrometer was placed on the motor-base-plate and the distance to the transducer-base-plate measured with an accuracy of $0.5 \mu \mathrm{m}$ during the increasing of the normal force. For all three instruments, the mechanically measured $K_{\mathrm{A}}$ is lower by about $20-30 \%$. For one rheometer, $K_{\mathrm{A}}$ has only to be determined once. Therefore, some effort should be made. Beyond the assumption of the validity of the simple viscoelastic oneparameter model, both methods described make additional assumptions. For the rheological method, a true compliantfree profile for $N_{1}(t)$ has assumed to be known (usually from simulation) to extract $K_{\mathrm{A}}$ from the best fit of Eq. (3). The same model, Eq. (3), is also applied in the correction of the measured normal force data. For the second method, it is assumed that the instrument deformation upon increasing the load in plate-plate contact mode is the same as the one resulting from the normal force of a sheared sample. The second method is certainly less time consuming and the stiffness can be determined with higher accuracy. Furthermore, it does not rely on a priori knowledge of the reference curve $N_{1}(t)$. Despite the still underlying assumptions, the method gives good results (see Fig. 7). This path to correct normal force data therefore will probably be the one preferred for many users.

\section{Relaxation after steady shear flow}

The simulation data $N_{1}$ shows that 15 shear units are sufficient to reach the steady state. The relaxation of $N_{1}(t>15 \mathrm{~s})$ can thus be treated as relaxation after steady shear flow, although $N_{1 \text {,meas. }}$ of the stiff rheometers RMS 800 and MCR 300 already shows an upturn due to starting edge fracture (see photographs Fig. 3). Whereas the torque relaxation $\eta_{\text {meas. }}(t) / \eta_{\text {meas. }}(t=15 \mathrm{~s})$ shows very weak depen-
Fig. 7 Correction of normal stress data measured on a rheometer of finite stiffness. Only experiments with a cone angle of $0.15 \mathrm{rad}$ on the ARES LR2 and the RMS 800 are shown. The sample radii are given in the legend

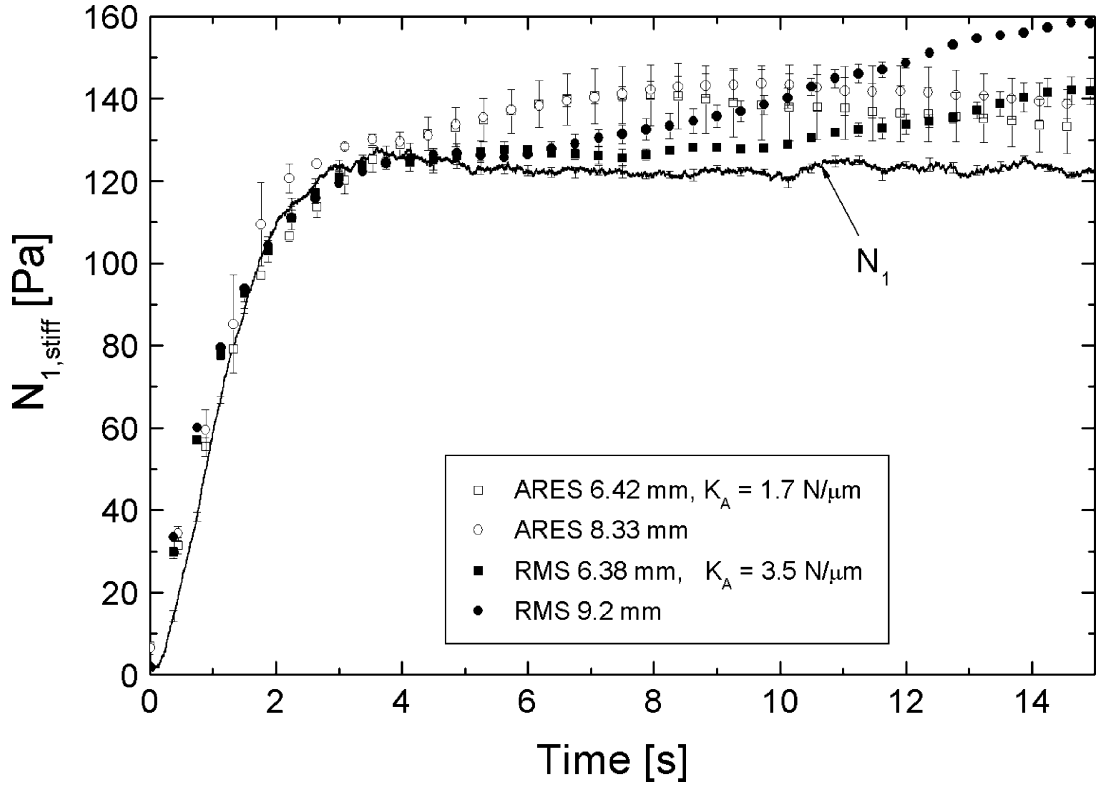


Fig. 8 a Characteristic torque decay time $\lambda_{\eta}^{\prime}\left(R, \alpha, K_{\mathrm{A}}\right)$, b characteristic normal force decay time $\lambda_{N I}^{\prime}\left(R, \alpha, K_{\mathrm{A}}\right)$ after steady shear flow for $15 \mathrm{~s}$ at $1 \mathrm{~s}^{-1}$. The line is a guide to the eye, with a $\lambda_{N I}^{\prime}=4 \cdot t_{\mathrm{a}}$. The axial response time $t_{\mathrm{a}}$ is calculated from Eq. 1. The open circles are experiments performed with the cone-partitioned plate (CCP) geometry. Note that the decay times in Fig. 8a are plotted on a linear, in Fig. 8b, on a log scale
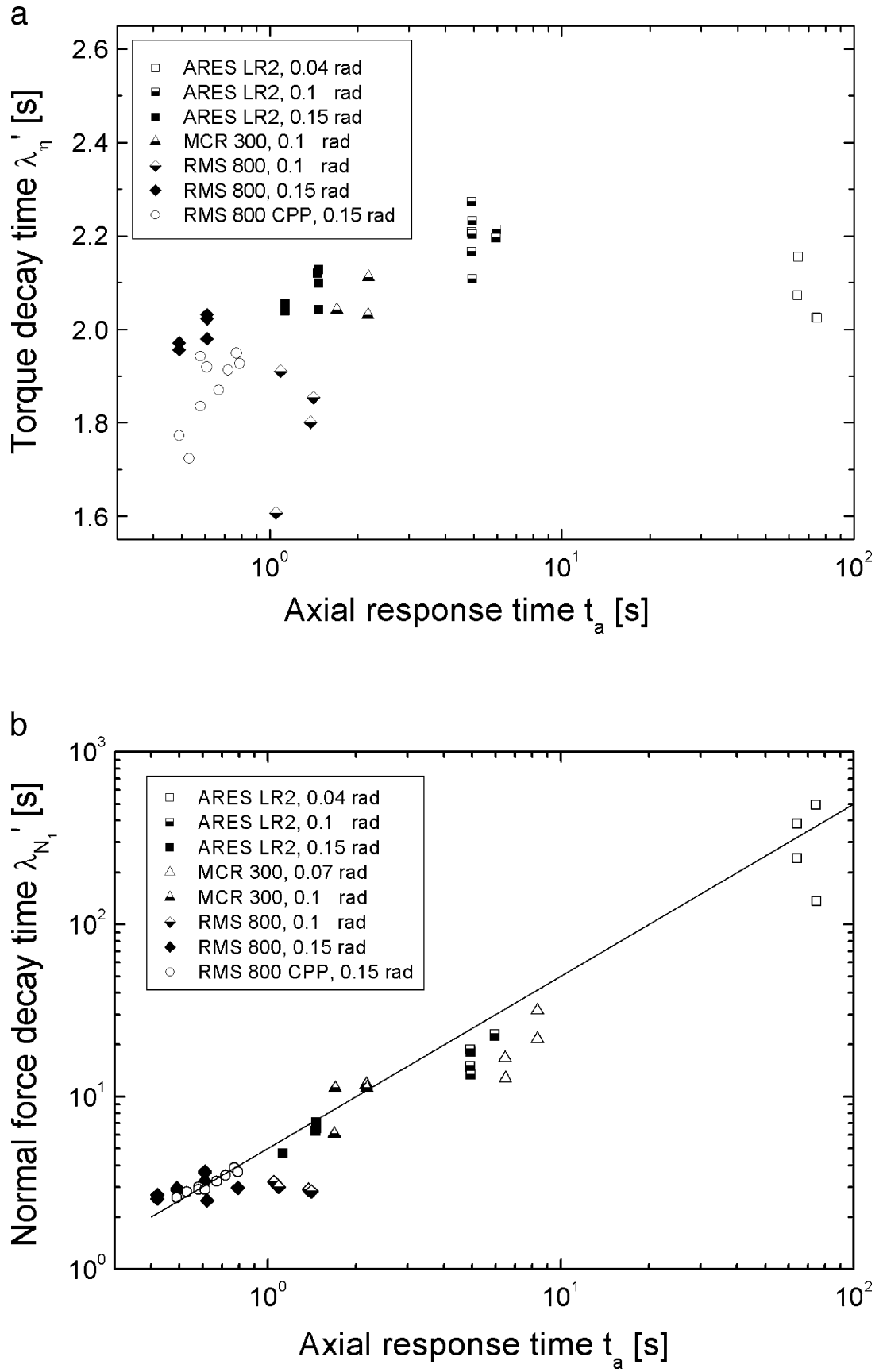

dence on geometrical parameters, $N_{1, \text { meas. }}(t) / N_{1 \text {,meas. }}(t=15 \mathrm{~s})$ strongly depends on experimental conditions $\left(R, \alpha, K_{\mathrm{A}}\right)$. From the linear part of the plots $\ln \left[\eta_{\text {meas. }}(t) / \eta_{\text {meas. }}(t=15 \mathrm{~s})\right]$ vs. $t$ and $\ln \left[N_{1 \text {,meas. }}(t) / N_{1, \text { meas. }}(t=15 \mathrm{~s})\right]$ vs. $t$, characteristic decay times $\lambda_{\eta}^{\prime}$ and $\lambda_{\mathrm{N}_{1}}^{\prime}$ were extracted (usually in the time window $16.5<t<22 \mathrm{~s}$ ) and plotted versus the axial response time $t_{\mathrm{a}}$ in Fig. 8a,b. The symbol $\lambda^{\prime}$ is used because this relaxation time is a mix of the rheometer's and the sample's properties. Figure $8 \mathrm{a}$ shows, that $\lambda_{\eta}^{\prime}$ only very moderately depends on $t_{\mathrm{a}}$. The scattering of the data with the same symbols is due to the fact that for the according curves, $\ln \left[\eta_{\text {meas. }}(t) / \eta_{\text {meas. }}(t=15 \mathrm{~s})\right]$ vs. $t$ does not show a neat linear part and the determination of $\lambda_{\eta}^{\prime}$ depends on the selection of the margins for the linear fit. Therefore, these margins were strictly set at $t=16.5$ and $22 \mathrm{~s}$ for all evaluations. Figure $8 \mathrm{~b}$ shows that even for the smallest $t_{\mathrm{a}}$ (RMS 800, $\alpha=0.15 \mathrm{rad}$, small $R) \lambda_{\mathrm{N}_{1}}^{\prime}$ does not yet approach a plateau value, as one might expect when the squeeze flow no longer makes a significant contribution to the relaxation process. Given the shape of the samples after 15 shear units at the beginning of 
the relaxation (Fig. 3), the scattering of the data is remarkably small. The open circles in Fig. $8 \mathrm{~b}$ are for the CPP geometry. Also for these experiments the measured decay time $\lambda_{\mathrm{N}_{1}}^{\prime}$ depends on sample size, thus clearly showing that the melt pressing on the fixed ring of the tool also makes its contribution to the widening of the instrument frame.

Samples of polystyrene of narrow molecular weight distribution are easily recovered after the experiment as these melts are not very tacky. Figure 3 shows the dilemma the rheologist is constantly faced with. Whereas a small sample squeezed below a 0.04 -rad cone perfectly keeps its shape for 15 shear units, the ones measured with the large cone of $0.15 \mathrm{rad}$ already start to form radial rolls. In spite of this observation in favor of a small cone angle, the experimental normal force data in Fig. 4 clearly forbids the use of small cone angles. One has to work with the large ones and take into account that at high shear rates the sample fractures before eventually reaching the steady state.

\section{Correction of time delay}

Equation (3) may be used to correct measurements for the time lag due to rheometer compliance. $N_{1}$ is then termed $N_{1, \text { stiff }}$ and is the function to be determined. This is an inverse problem for a Volterra equation of the first kind which are often not too severely ill-posed (Press et al. 1992). Still, a regularization method (Hansen 1998) is employed here to obtain stable estimates. A simple trapezoidal rule is sufficient for the discretization of Eq. (3) because the error is usually strongly dominated by the measurement noise.

Tikhonov regularization (see e.g. Hansen 1998) is used to determine an estimate $N_{1, \text { stiff }}$ from the measured data $N_{1 \text {,meas. }}$. Here, a term penalizing the curvature of the estimated function is added to the least squares criterion with the objective to ensure smooth solutions. The weighting factor of this smoothness penalty term, the so-called regularization parameter, is determined here using the heuristic Lcurve criterion (Hansen 1998). The computations were performed in Matlab using routines from the Regularization toolbox (Hansen 1994).

Due to finite compliance of the instrument, Eq. (3) shows that a normal stress increment at time $t$ is only fully expressed at a time $t^{\prime}>t$. $t^{\prime}-t$ depends on the experimental parameters in the same way as $t_{\mathrm{a}}$. If $t^{\prime}-t=t_{\mathrm{a}}, 63 \%$ of the normal stress increment is evolved. In the opposite way, if one wants to correct experimental data, information at time $t^{\prime}$ is needed to calculate the correct stress at time $t$. From this follows that if a certain shear rate is maintained up to time $t^{\prime}$, an accurate correction can only be made up to time

$t_{\max } \approx t^{\prime}-t_{\mathrm{a}}$.
The event at time $t^{\prime}$ can be a change in shear rate (e.g. start of relaxation) or simply the onset of edge fracture. From Eq. (7) follows immediately that a correction is most reasonable for $\alpha=0.15 \mathrm{rad}$. For this cone angle, $t_{\mathrm{a}}$ is sufficiently small compared to $15 \mathrm{~s}$, the persistence of the constant shear rate. Figure 7 shows the correction applied to the experiments performed with the largest cone angle of $0.15 \mathrm{rad}$ on the ARES LR2 and the RMS 800 with the $K_{\mathrm{A}}$ from mechanical contact measurements. If the 20 $30 \%$ larger $K_{\mathrm{A}}$ from rheology is used, there is no substantial difference except that $N_{1 \text {,stiff }}$ in the range $2<t<3 \mathrm{~s}$ is a bit lower than $N_{1}$. The data shows that edge fracture is a more severe restriction than Eq. (7) for the limit of validity of $N_{1, \text { stiff. }}$

\section{Summary and conclusion}

The time delay of normal force data $N_{1, \text { meas. }}$ measured relative to a compliance-free reference $N_{1}$ is due to the finite stiffness of commercial rheometers. If the rheometer stiffness $K_{\mathrm{A}}$ is known, $N_{1, \text { meas. }}$ can be corrected. $K_{\mathrm{A}}$ can be obtained from mapping $N_{1}$ on $N_{1 \text {,meas. }}$ or from direct mechanical plate-plate contact measurements without sample. The first method involves the assumption of a simple viscoelastic model and the knowledge of compliantfree reference data. The second method assumes that further squeezing the plates in contact deforms the instrument in the same way as the normal force of the sheared sample. The discrepancy of $20-30 \%$ in $K_{\mathrm{A}}$ obtained with the two methods indicates that the viscoelastic model is probably too simple. However, for the correction of $N_{1 \text {, meas. }}$, the two values of $K_{\mathrm{A}}$ both give quite satisfactory results. It has to be mentioned that the $20-30 \%$ scatter in $K_{\mathrm{A}}$ is much smaller than the range of values found in literature. A ranking of rheometer stiffnesses can be made and those experimental conditions identified which are less prone to false $N_{1}$ measurements.

The characteristic delay time obtained from normal force relaxation after steady shear flow scales linearly with the axial response time $t_{\mathrm{a}}$ of the rheometer calculated from $K_{\mathrm{A}}$, cone angle, and sample radius. This is important to consider when doing nonlinear stress relaxation or large amplitude oscillatory (LAOS) experiments. Luckily, the torque relaxation time is almost independent of $t_{\mathrm{a}}$.

This work shows an old dilemma in rheology. While a 0.04-rad cone gives reproducible $N_{1 \text {,meas. }}$-data, correct viscosities and almost no edge fracture after 15 strain units, the absolute value of $N_{1 \text {,meas. }}$ is completely off and $N_{1 \text {,meas. }}$ relaxation times can be almost two orders of magnitude too high. For correct $N_{1 \text {,meas. }}$-data, a cone angle of $0.15 \mathrm{rad}$ has to be used, for which edge fracture already sets in after roughly 5-10 strain units and with which the determination of reproducible viscosity and $N_{1 \text {,meas. }}$ steady state values is more difficult. 
The only way out of this dilemma is to work with solutions or to use the CPP technique, which is currently limited by the insufficient normal force capacity of the transducers and also not commercially available.

This report is for a melt with narrow molecular weight distribution. Normal force measurements have also been previously performed with a technical polystyrene melt (PS $158 \mathrm{~K}$, Schweizer 2002). For this melt, transient normal stress data was less affected by compliance. It was not within the scope of this paper to investigate how the width of the molecular weight distribution affects the transient behavior and whether the model, Eq. (3), is equally powerful for the correction of the measured data. From the observations made in Schweizer et al. 2004 concerning the stability of the flow, it might be expected however that the data in Figs. 4 and 5 for the largest cone angle would show a better reproducibility in the steady state.

The results of this investigation also show that frame stiffnesses of well-known instruments often cited in literature are not necessarily correct. It was found for the RMS800 that the effective axial compliance is a factor of four lower than cited in previous papers (Meissner et al. 1989; Schweizer et al. 2004).

Acknowledgements The authors would like to thank Jan van Meerveld for the simulation of the first normal stress difference data and Simon Aggeler, Esther Amstad, Barbara Grant, and Marina Karlina for the help with the experiments. A.B. gratefully acknowledges financial support by the Deutsche Forschungsgemeinschaft through grant BA 2884/1-1.

\section{References}

Fetters LJ, Lohse DJ, Richter D, Witten TA, Zirkel A (1994) Connection between polymer molecular weight, density, chain dimension, and melt viscoelastic properties. Macromolecules 27(17):4639-4647

Gleissle W (1976) Pressure distribution in gap of cone and plate rheometer with respect to shear of viscoelastic liquids having high shear rates. Rheol Acta 15 (6):305-316 (in German)

Gleissle W (1978) A cone-plate rheometer for highly viscous viscoelastic fluids at high shear rates, investigation of the flow behavior of high molecular silicon oil and poly(isobutylene). Thesis, University of Karlsruhe (in German)

Gleissle W (1980) Two simple time-shear rate relations combining viscosity and first normal stress coefficient in the linear and non-linear flow range. In: Astarita $\mathrm{G}$ et al (eds) Rheology, vol 2. Plenum, New York, pp457-462

Gleissle W, Ohl N (1990) On the relaxation of shear and normal stresses of viscoelastic fluids following constant shear rate experiments. Rheol Acta 29:261280
Hansen PC (1994) Regularization Tools: A Matlab package for analysis and solution of discrete ill-posed problems. Numer Algorithms 6:1-35

Hansen PC (1998) Rank-deficient and discrete ill-posed problems: numerical aspects of linear inversion. SIAM, Philadelphia

Hansen MG, Nazem F (1975) Transient normal force transducer response in a modified Weissenberg rheogoniometer. Trans Soc Rheol 19(1):21-36

Kaye A, Lodge AS, Vale DG (1968) Determination of normal stress differences in steady shear flow. II. Flow birefringence, viscosity, and normal stress data for a polyisobutylene liquid. Rheol Acta 7(4):368-379

Likhtman AE, McLeish TCB (2002) Quantitative theory for linear dynamics of linear entangled polymers. Macromolecules 35:6332-6343

Lodge AS, Meissner J (1972) On the use of instantaneous strains, superposed on shear and elongational flows of polymeric liquids, to test the Gaussian network hypothesis and to estimate the segment concentration and its variation during flow. Rheol Acta 11:351-352

Meissner J (1972) Modification of the Weissenberg Rheogoniometer for measurement of transient rheological properties of molten polyethylene under shear. Comparison with tensile data. J Appl Polym Sci 16:2877-2899
Meissner J, Garbella RW, Hostettler J (1989) Measuring normal stress differences in polymer melt shear flow. J Rheol 33 (6):843-864

Öttinger HC (1999) A thermodynamically admissible reptation model for fast flows of entangled polymers. J Rheol 43:1461-1493

Press WH, Teukolsky SA, Vetterling WT, Flannery BP (1992) Numerical recipes in $\mathrm{C}$ - the art of scientific computing. 2nd edn. Cambridge University Press, p 790

Schweizer T (2002) Measurement of the first and second normal stress differences in a polystyrene melt with a cone and partitioned plate tool. Rheol Acta 41:337-344

Schweizer T, van Meerveld J, Öttinger HC (2004) Nonlinear shear rheology of polystyrene melt with narrow molecular weight distribution - experiment and theory. J Rheol 48(6):1345-1363

Schweizer T (2005) Temperature calibration of rotational rheometers with electrically heated tools and hood oven. Appl Rheol 15(2):113-117 